\title{
Öffentliches Experiment
}

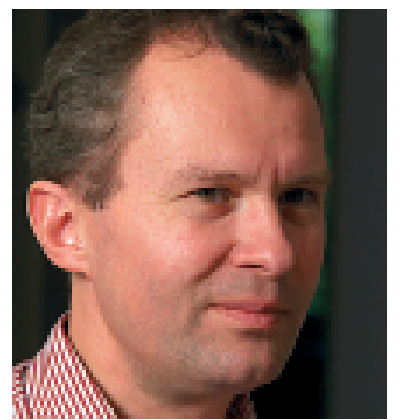

* Prof. Dr. phil., dipl. biol. Christoph Rehmann-Sutter leitet die Arbeitsstelle für Ethik in den Biowissenschaften der Universität Basel, ist Präsident der Nationalen Ethikkommission und Mitglied der Redaktion Ethik der Schweizerischen Ärztezeitung.
Wie kann man verhindern, dass wissenschaftliche Publikationen fabrizierte Daten enthalten? Die komplette Demontage der südkoreanischen Stammzellenforschung wirft unter anderem diese Frage auf. Auch wenn eine Information von den anerkanntesten unserer wissenschaftlichen Wahrheitsfilter (high-impact journals) nicht aussortiert wurde, muss sie deshalb nicht schon wahr sein. Das wäre zwar eigentlich evident, kann doch jeder wissenschaftliche Satz nur eine Hypothese sein, die noch nicht falsifiziert worden ist. Gehört aber eine Untersuchungskommission der eigenen Universität, die eingesetzt wurde, um Betrugsbehauptungen zu überprüfen, zum normalen Popperschen Prozedere der Falsifikation von Hypothesen?

Poppers Theorie ist zu einfach. Die beiden fraglichen Science-Papers von Dr. Woo Suk Hwang et al. enthielten nebst theoretischen auch viele andersartige Sätze: Sätze darüber, dass man das Experiment des therapeutischen Klonens erfolgreich durchführen konnte, dass es gelang, 11 Stammzellinien zu entwickeln, die genetisch mit den Patientinnen und Patienten übereinstimmen usw. Oder Sätze darüber, was auf «beweisenden» Fotografien zu sehen ist, die sich jetzt als Fabrikationen entpuppt haben. Sätze dieser Art sind gar keine theoretischen Sätze im Sinne Poppers. Sie können nicht als Hypothesen aufgefasst werden, sondern müssen stimmen, damit die Hypothese, der die Publikation gilt, überhaupt glaubhaft wird.

Es ist ja so, dass die Leser eines wissenschaftlichen Journals den Berichten, die bestimmte Hypothesen glaubwürdig machen, Vertrauen schenken müssen. Sie können nicht ins betreffende Labor gehen und sich die Sache selbst ansehen. Eine Form von Glauben gehört zum Alltagsgeschäft der Wissenschaft: der Glaube an die Authentizität des Berichteten, auch wenn man die darauf basierende theoretische Konklusion, also die eigentliche Hypothese, vielleicht in Zweifel zieht.

Nach den Enthüllungen vom 9. Januar 2006 in Seoul wurde viel debattiert über die Trennschärfe von Peer-review-Verfahren und auch darüber, wie ein so gigantischer Betrug überhaupt gelingen konnte. Dr. Hwang hatte ja Mitarbeiterinnen und Mitarbeiter, ein grosses internationales Team. Wie konnten all diese Leute involviert werden? Was wussten sie? Was nicht? Wie konnten sie getäuscht werden? Wie weit reicht Loyalität in wissenschaftlichen Instituten? Und - für mich immer noch rätselhaft - wie konnte eine Koryphäe, die einen Ruf zu verlieren und Verantwortung für viele Mitarbeiter hatte, so etwas wagen, das doch absehbar einmal auffliegen musste?

Vielleicht können wir eine Lehre aus dem Vorgang ziehen, nämlich die, dass für den normalen Fortgang der Spitzenforschung etwas ganz Einfaches und ganz Menschliches, nämlich Vertrauen notwendig ist. Der Glauben, den die Leserinnen und Leser den Berichten schenken müssen, die in einem Journal publiziert werden, hat seine Grundlage darin, dass sie Vertrauen setzen in die Vertrauenswürdigkeit der Autorschaft und in die Prüfung der Zuverlässigkeit der angegebenen Belege durch ein Reviewverfahren. Die Peers, die sich an einem Review beteiligen, müssen ebenfalls Vertrauen setzen in die Wahrhaftigkeit der Aussagen der Autorschaft. Sie können nur selten unabhängige Evidenzen auf derselben Ebene herbeiholen oder das Experiment selbst im Labor nachkontrollieren.

Wie funktioniert die Forschung auf dieser menschlichen Ebene, die für das Vertrauen konstitutiv ist? Es ist offensichtlich nicht möglich, Vertrauen zu schaffen durch die Perfektionierung der Kontrollmechanismen; es bleibt ein Rest. Eigentlich war das eine medial vermittelte Variante eines öffentlichen Experiments, ähnlich wie im 18. Jahrhundert, als die Wissenschaftler ihre Versuche vor Publikum zeigten und damit ihre Glaubwürdigkeit unter Beweis stellten. Auch jene Experimente beruhten wesentlich auf Vertrauen. Das Publikum konnte zwar direkt mit eigenen Augen zusehen, musste aber davon ausgehen dürfen, dass die dargebotenen Effekte nicht getürkt waren. Vertrauen als ein uneinholbarer Rest, der für die Forschung konstitutiv ist: Diese Erkenntnis bietet einigen Stoff.

Christoph Rehmann-Sutter* 